\title{
The Effect of Notch Filter on RFI Suppression
}

\author{
Wenge CHANG, Jianyang LI, Xiangyang LI \\ School of Electronics Science and Engineering, National University of Defense Technology, Changsha, China \\ E-mail: changwenge@sina.com \\ Received May 22, 2009; revised May 31, 2009; accepted June 10, 2009
}

\begin{abstract}
Radio Frequency Interference (RFI) suppression is an important technique in the ultra-wideband synthetic aperture radar (UWB SAR). In this paper, we mainly analyze the performance of a notch filter for RFI suppression. The theoretical output from notch filter is presented based on RFI signal's narrowband property. The research conclusion shows that the notch filter has significant effect on sidelobes of the system response, which might be considered to be false targets, however it has little effect on the resolution of the system response. The theoretical result is verified by simulation and experimental data processing both in one dimension (range dimension) and in two dimensions (range and azimuth dimension).
\end{abstract}

Keywords: RFI Supression, Matched Filter, Notch Filter, SAR

\section{Introduction}

The dual requirement of a low radar frequency for foliage and/or ground penetration and a wide radar bandwidth for high resolution in wideband radar systems leads to radar operating in frequency bands occupied by other radio systems, such as TV and radio communications. As a result, Radio Frequency Interference (RFI) appears in the received radar signal [1-3]. In ultra-wideband synthetic aperture radar (UWB SAR), the RFI energy is spread over the whole image scene, displaying artefacts and masking targets, especially in low SNR areas [1-5]. Any subsequent processing in UWB SAR (such as target classification, interferometry, etc.) would be degraded by the presence of RFI. Thus, RFI suppression is an important technique in UWB SAR signal processing.

The common approach to RFI suppression is to examine the spectrum of the contaminated signal, identify the interference spikes which usually have greater power than the radar echo signal, and subsequently remove these spikes with the help of a notch filter [1]. The notch concept is effective for RFI suppression. Having studied the notch filter based on least-mean-squared estimation and tested with real RFI data, T. Koutsoudis and L. Lovas suggested that the notch filter can produce an adverse impact on the SAR performance (such as reducing image intensity, range resolution and creating loss in the target's signal to noise ratio) but no theoretic results were presented in reference to this claim [2].
In this study, the performance of the notch filter is theoretically analysed. Firstly, the transmitted radar signal model, the received radar signal model and the RF interference model are presented. A matched filter with notches is designed. Secondly, the contaminated signal (the sum of radar echo and RFI) is fed to the matched filter, and the theoretical output of the filter is derived. The theoretical result shows that the output of the matched filter with notches is influenced by the notches' width and carrier and, furthermore that the notch filter has little impact on range resolution but significant impact on sidelobes. The simulation is studied both in the case of one RF interference existing as well as multiple $\mathrm{RF}$ interference existing. Finally, the matched filter with notches is applied to the experimental data acquired by an airborne UWB SAR, and the validity of the theoretical result is tested.

This paper is arranged as follows: In part 2, the models are built and the output from the notch filter is theoretical deduced. In part 3 , the simulation in range is carried out and the result is shown to be consistent with theoretical result. In part 4, the SAR image simulation as well as the experimental UWB SAR image is processed in order to test and verify the validity of the theoretical result. Finally, the results are summarised in the conclusion.

\section{Modelling and Theoretical Deducing}

Two assumptions are made in order to make analysis 
more convenient. Firstly, it is assumed that the transmitted and received signal of UWB SAR is a base-band linear frequency modulated pulse as follows:

$$
\begin{gathered}
s(t)=\operatorname{rect}\left(\frac{t}{T}\right) e^{j \pi k^{2}}, k=B / T ; \\
S(\omega)=\operatorname{rect}\left(\frac{\omega}{2 \pi B}\right) e^{-j \frac{\omega^{2}}{4 \pi k}}
\end{gathered}
$$

where $B$ is the bandwidth, $T$ is the width of the transmitted pulse, $S(\omega)$ is the spectrum of $s(t)$.

Secondly, it is assumed that only one narrow band RFI signal exists with carrier $\omega_{1}$ and bandwidth $b_{1}$.

The matched filter with a notch at $\omega_{1}$ is designed for suppressing FRI with carrier $\omega_{1}$ and bandwidth $b_{1}$. The matched filter in spectrum is shown in Equation (3).

$$
H(\omega)=\left(1-\operatorname{rect}\left(\frac{\omega-\omega_{1}}{2 \pi b_{1}}\right)\right) S^{*}(\omega)=S^{*}(\omega)-\operatorname{rect}\left(\frac{\omega-\omega_{1}}{2 \pi b_{1}}\right) e^{j \frac{\omega^{2}}{4 \pi k}}
$$

The received radar signal includes the echo signal (the reflected transmitted signal from the target), the RFI signal and thermal noise. Assuming only one RFI $s_{R F I 1}(t)$ exists and that thermal noise can be ignored, the received signal $s_{r}(t)$, and its spectrum $S_{r}(\omega)$, can be written as

$$
\begin{gathered}
s_{r}(t)=a \cdot s(t-\tau)+s_{R F I 1}(t) \\
S_{r}(\omega)=a S(\omega) e^{-j \omega \tau}+S_{R F 11}(\omega)
\end{gathered}
$$

where $a$ is a constant coefficient. The received signal is processed with the matched filter. The output in spectrum is given by Equation (6).

$$
\begin{gathered}
Y(\omega)=S_{r}(\omega) H(\omega)=\left(a S(\omega) e^{-j \omega \tau}\right. \\
\left.+S_{R F I 1}(\omega)\right) \cdot\left(S^{*}(\omega)-\operatorname{rect}\left(\frac{\omega-\omega_{1}}{2 \pi b_{1}}\right) e^{j \frac{\omega^{2}}{4 \pi k}}\right) \\
=a \cdot \operatorname{rect}\left(\frac{\omega}{2 \pi B}\right) e^{-j \omega \tau}+S^{*}(\omega) S_{R F I 1}(\omega) \\
\quad-a S(\omega) e^{-j \omega \tau} \cdot \operatorname{rect}\left(\frac{\omega-\omega_{1}}{2 \pi b_{1}}\right) e^{j \frac{\omega^{2}}{4 \pi k}} \\
-a \cdot \operatorname{rect}\left(\frac{\omega-\omega_{1}}{2 \pi b_{1}}\right) e^{j \frac{\omega^{2}}{4 \pi k}} \cdot S_{R F I 1}(\omega)
\end{gathered}
$$

where:

$$
\begin{aligned}
& a S(\omega) e^{-j \omega \tau} \cdot \operatorname{rect}\left(\frac{\omega-\omega_{1}}{2 \pi b_{1}}\right) e^{j \frac{\omega^{2}}{4 \pi k}} \\
& \quad=a \cdot \operatorname{rect}\left(\frac{\omega}{2 \pi B}\right) e^{-j \frac{\omega^{2}}{4 \pi k}} \cdot e^{-j \omega \tau} \cdot \operatorname{rect}\left(\frac{\omega-\omega_{1}}{2 \pi b_{1}}\right) e^{j \frac{\omega^{2}}{4 \pi k}} \\
& =a \cdot \operatorname{rect}\left(\frac{\omega-\omega_{1}}{2 \pi b_{1}}\right) \cdot e^{-j \omega \tau}
\end{aligned}
$$

$$
S^{*}(\omega) S_{R F I 1}(\omega)=\operatorname{rect}\left(\frac{\omega}{2 \pi B}\right) e^{j \frac{\omega^{2}}{4 \pi k}} \cdot S_{R F I 1}(\omega)
$$

Thus:

$$
\begin{aligned}
& Y(\omega)=a \cdot \operatorname{rect}\left(\frac{\omega}{2 \pi B}\right) e^{-j \omega \tau}-a \cdot \operatorname{rect}\left(\frac{\omega-\omega_{1}}{2 \pi b_{1}}\right) e^{-j \omega \tau} \\
& \quad+\operatorname{rect}\left(\frac{\omega}{2 \pi B}\right) e^{j \frac{\omega^{2}}{4 \pi k}} \cdot S_{R F I 1}(\omega)-\operatorname{rect}\left(\frac{\omega-\omega_{1}}{2 \pi b_{1}}\right) e^{j \frac{\omega^{2}}{4 \pi k}} \cdot S_{R F I 1}(\omega)
\end{aligned}
$$

Based on the assumption that the RFI signal $s_{R F I 1}(t)$ is a narrow band signal with carrier $\omega_{1}$ and bandwidth $b_{1}$, we have

$$
\operatorname{rect}\left(\frac{\omega}{2 \pi B}\right) e^{j \frac{\omega^{2}}{4 \pi k}} \cdot S_{R F I 1}(\omega) \approx \operatorname{rect}\left(\frac{\omega-\omega_{1}}{2 \pi b_{1}}\right) e^{j \frac{\omega^{2}}{4 \pi k}} \cdot S_{R F I 1}(\omega)
$$

Considering Equations (10) and (9), the output of the matched filter with a notch is given by

$$
Y(\omega)=a \cdot \operatorname{rect}\left(\frac{\omega}{2 \pi B}\right) e^{-j \omega \tau}-a \cdot \operatorname{rect}\left(\frac{\omega-\omega_{1}}{2 \pi b_{1}}\right) e^{-j \omega \tau}
$$

The output in the time domain of the matched filter with a notch is given by

$$
y(t)=a B \cdot S a(B(t-\tau))-a b_{1} \cdot S a\left(b_{1}(t-\tau)\right) e^{-j \omega_{1}(t+\tau)}
$$

From Equation (12) we find that the output of the matched filter with a notch has two parts: one is the desired part and the other one is the undesired part. Here we call the undesired part clutter, which is a high frequency oscillating signal modulated by a wide pulsed sinc-function, determined by parameters $\omega_{1}, b_{1}$. This clutter influences the peak-sidelobe-ratio (PSLR), the integrated-sidelobe-ratio (ISLR) of radar system response.

Here the signal-to-clutter-ratio $(S C R)$ is utilised to describe the influence as follows:

$$
S C R=20 \log \left(b_{1} / B\right)
$$

It is easy to expand Equation (12) for multiple RF interference signals. Assuming there are $n$ RFI signals with carriers and bandwidths being $\omega_{n}, b_{n}(n=1,2, \ldots, n)$ respectively.

The matched filter with $n$ notches is given by:

$$
\begin{aligned}
H(\omega) & =\left(1-\sum_{k=1}^{n} \operatorname{rect}\left(\frac{\omega-\omega_{k}}{2 \pi b_{k}}\right)\right) S^{*}(\omega) \\
& =S^{*}(\omega)-\sum_{k=1}^{n} \operatorname{rect}\left(\frac{\omega-\omega_{k}}{2 \pi b_{k}}\right) \cdot e^{j \frac{\omega^{2}}{4 \pi k}}
\end{aligned}
$$

The output spectrum of the matched filter is: 
$Y(\omega)=a \cdot \operatorname{rect}\left(\frac{\omega}{2 \pi B}\right) e^{-j \omega \tau}-a \cdot \sum_{k=1}^{n} \operatorname{rect}\left(\frac{\omega-\omega_{k}}{2 \pi b_{k}}\right) e^{-j \omega \tau}$

Corresponding time domain expression is:

$y(t)=a B \cdot S a(B(t-\tau))-a \cdot \sum_{k=1}^{n} b_{k} \cdot S a\left(b_{k}(t-\tau)\right) e^{-j \omega_{k}(t+\tau)}$

For $n$ RFI signals, the output of the matched filter with $n$ notches also consists of two parts: one is desired, the other one is undesired (which is the sum of $n$ high frequency oscillating signal modulated by wide pulsed sinc-function, determined by parameters $\omega_{n}, b_{n} n=1$, $2, \ldots, n)$

$S C R$ is no longer suitable for describing the influence of the notch filter under these conditions. For the clutter, being coherent, might accumulate, some of the sidelobes would be higher than the case when only one RFI signal exists.

From the Equation (12) and Equation (16), we have the conclusion that the notch filter affects the output of matched filter in following ways:

- It produces the undesired output that is an oscillating signal modulated by a sinc-function. The undesired output influences PSLR and ISLR of radar system response.

- It has little influence on the resolution of radar system response.

\section{Simulation}

We have two simulation steps in order to demonstrate the validity of Equation (16) with only one RFI and multiple RFI signals existing.

\subsection{Only One RFI Existing}

It is assumed that the base-band LFM signal has 20us pulse-width with $200 \mathrm{MHz}$ bandwidth, and that the RFI carrier is $30 \mathrm{MHz}$ and the bandwidth is $8 \mathrm{MHz}$. Under the above conditions, the spectrum of the matched filter with a notch is shown in Figure 1. The output of the matched filter with a notch is shown in Figure 2. In the figure the ideal output, the output of the ideal matched filter for a ideal LFM input, is as dotted line and the output of the matched filter with RFI suppression for the mixed signal is as solid line. Comparing two outputs we find that regular spikes appear in the output with RFI suppression.

To describe the effect of the notch filter in a clear way, the output is separated two parts, as the Equation (12), described as in Figure 3. The solid line is the desired output and the dotted line is undesired part. From the figure, the $S C R$ is about $-27.6 \mathrm{~dB}$, being consistent with the theoretical result of $-27.96 \mathrm{~dB}$ derived from Equation (13).

Figure 4 is the output of ideal matched filter for mixed signal. From the figure, the sidelobes rise, esp., the sidelobes being far from the mainlobe, as a result the clutter rise greatly.

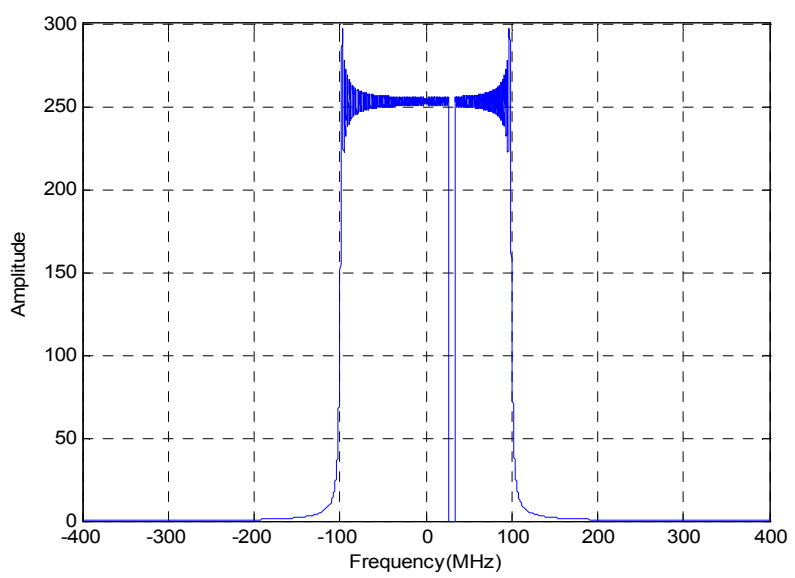

Figure 1. Matched filter with a notch.

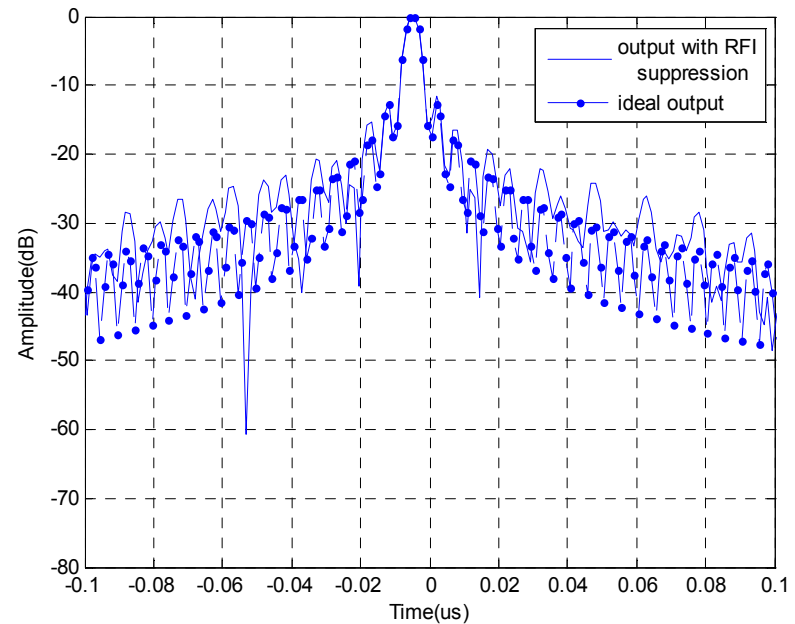

Figure 2. The output of matched filter.

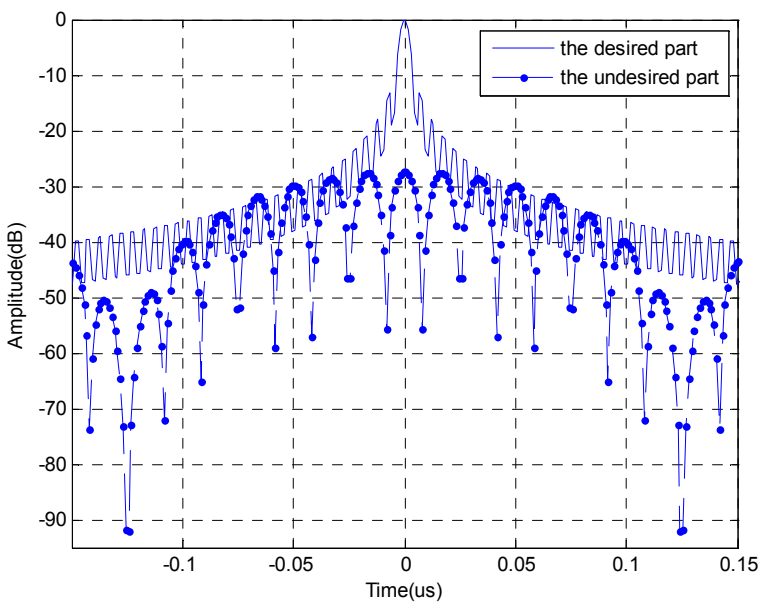

Figure 3. The separated form of output. 


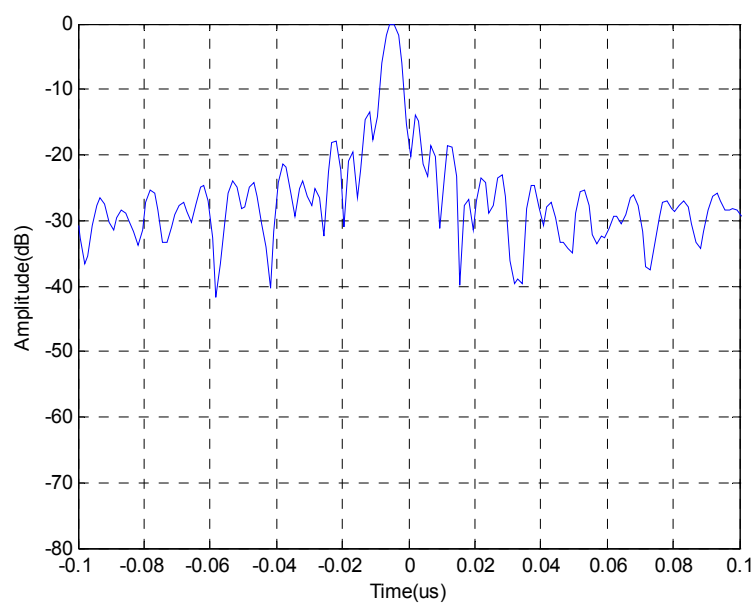

Figure 4. The output for mixed signal.

From the figures we find that the notch filter do have significant effect on RFI suppression, however, it has side effect that the sidelobes raise greatly.

\subsection{Multiple RFI Existing}

It is assumed that the base-band LFM signal is as same as above. Meanwhile, three existing RFI signals are assumed and the RFI signals' carriers and bandwidths are $(-50 \mathrm{MHz}, 8 \mathrm{MHz}),(30 \mathrm{MHz}, 8 \mathrm{MHz})$, and $(50 \mathrm{MHz}, 8 \mathrm{MHz})$ respectively. Under these conditions, the spectrum of the matched filter with notches is shown in Figure 5.

The output of the matched filter with notches is shown in Figure 6. In the figure the ideal output is as dotted line and the output of the matched filter with RFI suppression for the mixed signal is as solid line. Comparing two outputs we also find that regular spikes appear in the output with RFI suppression.

The output is also separated two parts, as the Equation

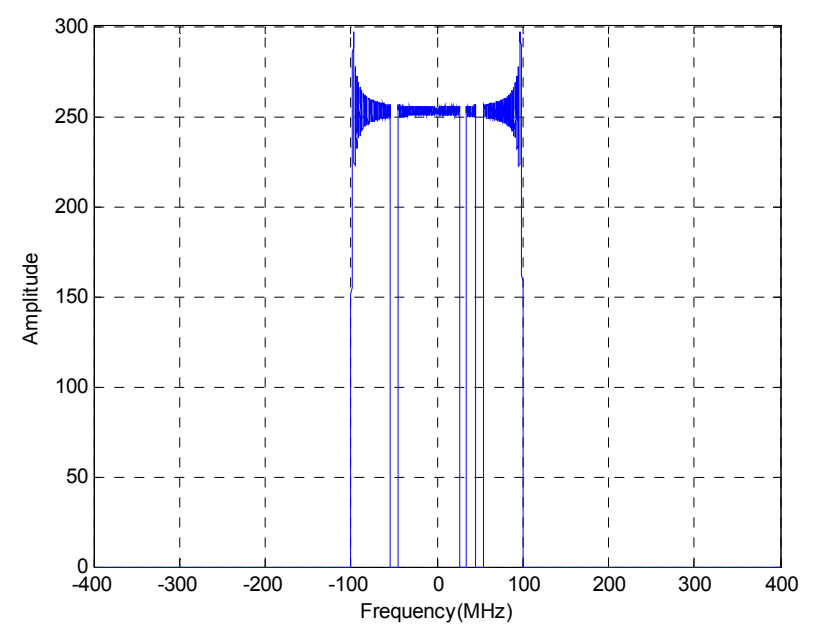

Figure 5. Matched filter with notches.

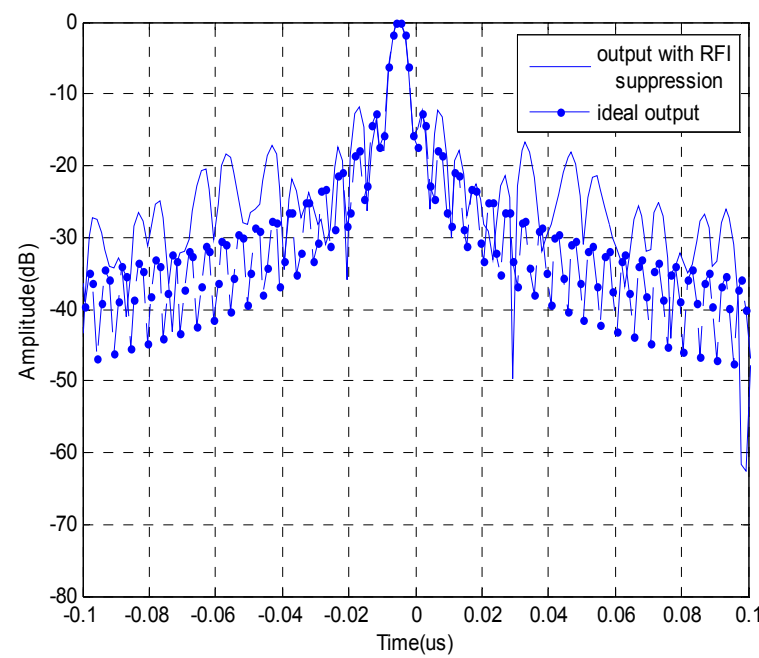

Figure. 6 The output of matched filter with notches.

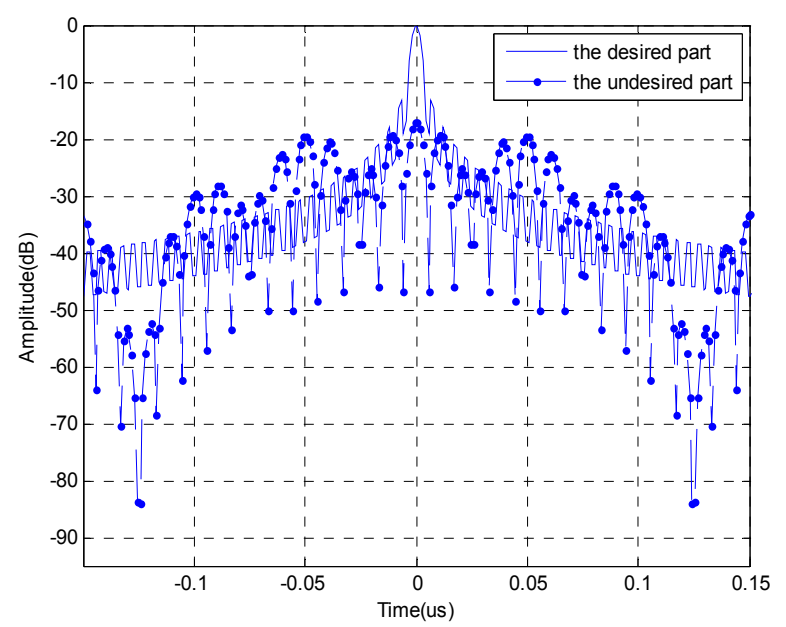

Figure 7. The separated form of output.

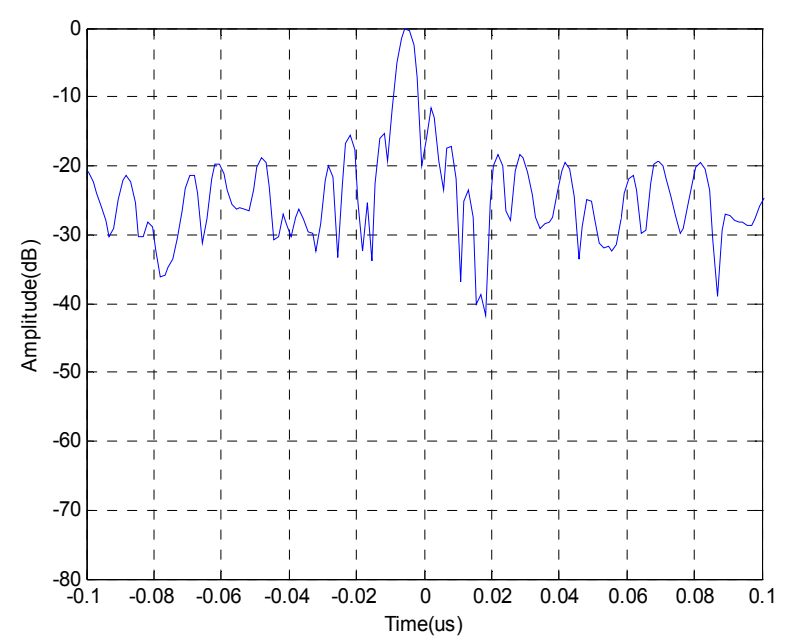

Figure 8. The output for mixed signal. 
(16), described as in Figure 7. It can be observed that the $S C R$ is significantly higher than in the case of only one existing RFI. We measure the highest $S C R$ as $-18.2 \mathrm{~dB}$, which is consistent with the theoretical result $20 \operatorname{Lg}(3 b / B)=-18.42 d B$ derived from Equation (13).

Figure 8 is the output of ideal matched filter for mixed signal. From the figure, we have the same conclusion as the former: sidelobes rise, esp., the sidelobes being far from the mainlobe, causing the clutter rise greatly.

We also find that the notch filter do have significant effect on RFI suppression, however, it has side effect. In summary, the notch filter has significant effect on RFI suppression, but it can produce an adverse impact that the sidelobes rise on the output of the matched filter. Meanwhile we find that notch filter has little impact on range resolution.

\section{Real Data Processing}

To check the validity of the theoretical conclusion we apply the matched filter with notch(s) to the real data

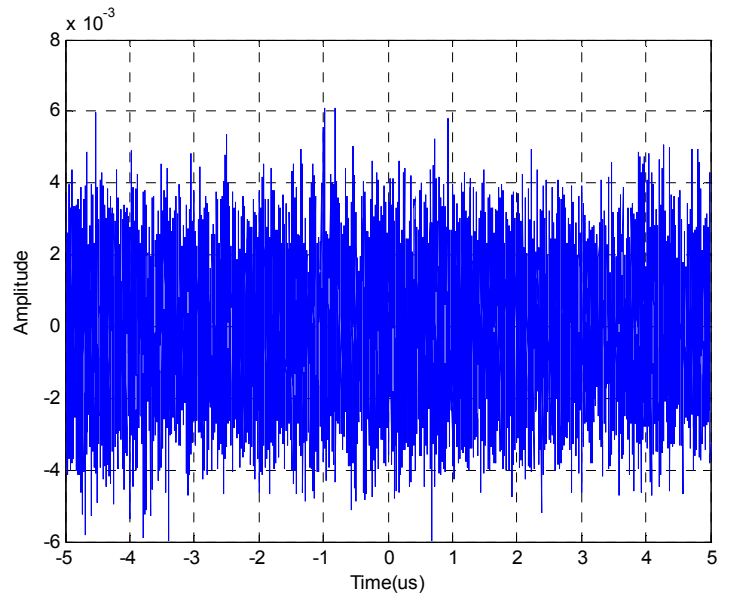

Figure 9. The received signal.

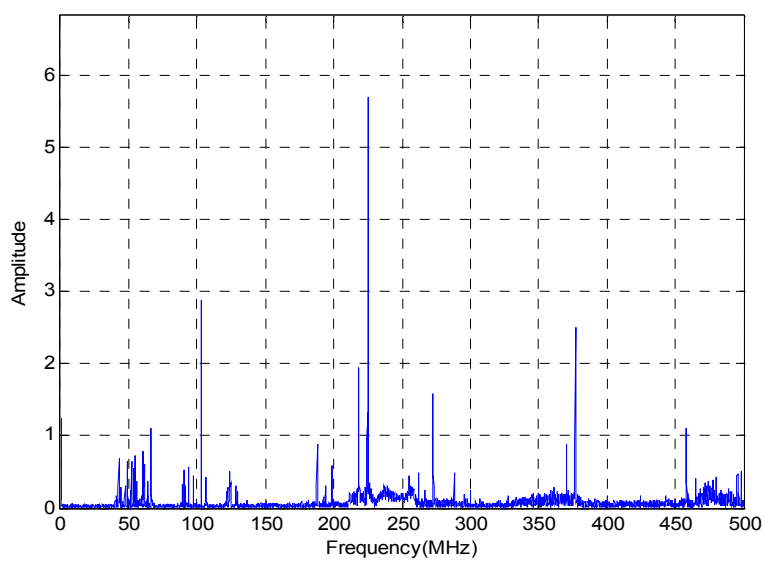

Figure 10. The spectrum of received signal. from a specified system. In the system, the transmitted signal, which is LFM signal with 10us pulse-width, $50 \mathrm{MHz}$ bandwidth and $235 \mathrm{MHz}$ centre frequency, is generated by AWG520. This signal is fed to an antenna which is Archimedes screw form to radiation. Meanwhile a same form antenna is used to receive. The distance between two antennas is $8 \mathrm{~m}$. The received signal is amplified, and then is sampled by TDS784D Digital Oscilloscope. The received signal is as Figure 9. The spectrum is as Figure 10. From the Figures, we can see there are two RFI signals in the bandwidth of $[210 \mathrm{MHz}$ $260 \mathrm{MHz}$, but only one RFI is relatively strong. So we use the notch filter to suppress this stronger one. The notch filter's carrier and bandwidth are $221.5 \mathrm{MHz}$ $6.5 \mathrm{MHz}$ respectively.

The output of matched filter without notch is shown in Figure 11. It is clear that RFI has intense influence that sidelobes, whatever are far or near from mainlobe, are raised to about $-13 \mathrm{~dB}$ on the system response.

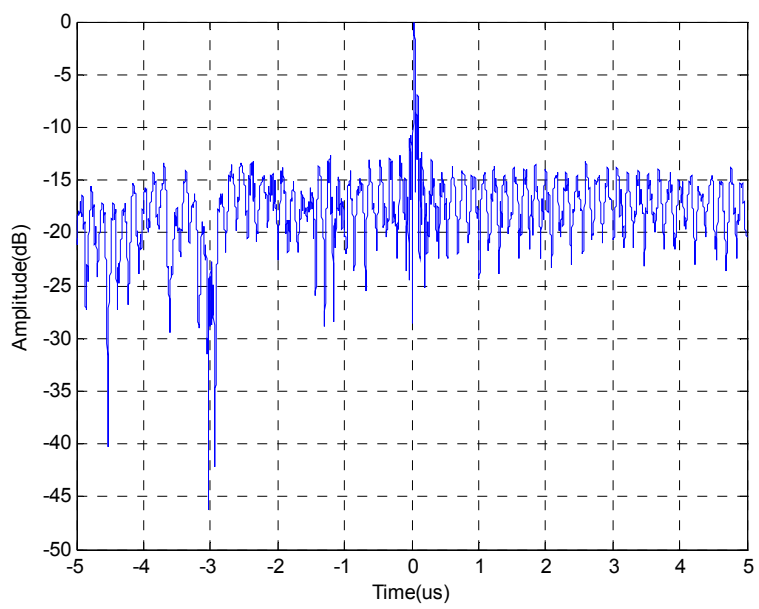

Figure 11. The output of matched filter without notch.

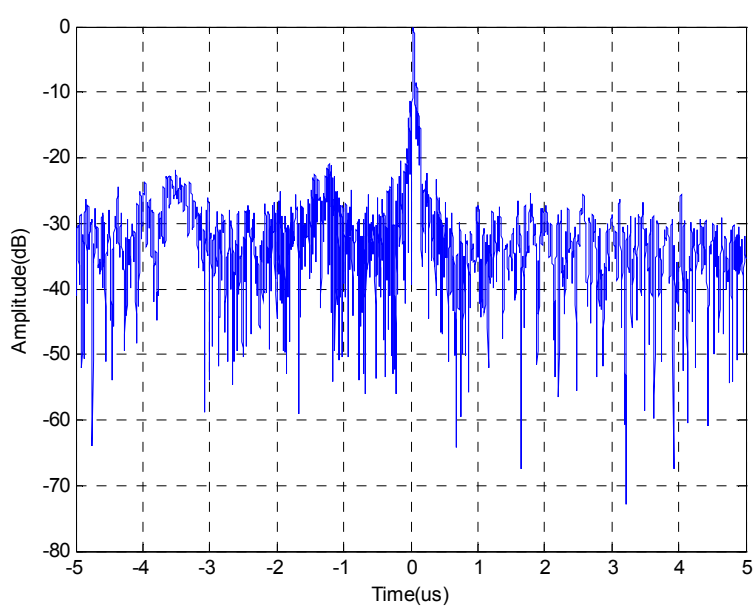

Figure 12. The output of matched filter with notch. 


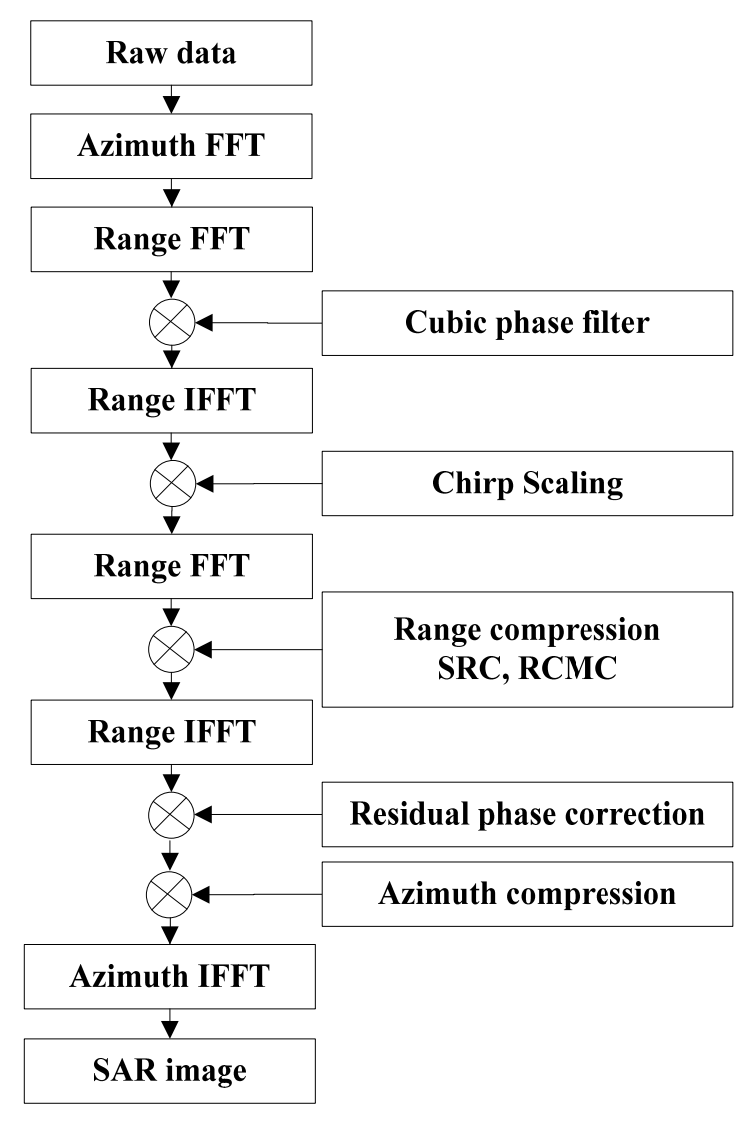

Figure 13. The diagram of NCS image algorithm.

The output of matched filter with notch is shown in Figure 12. It can be seen that the influence of RFI have been decreased greatly, and the sidelobes level is less than $-21 \mathrm{~dB}$. However, the regular spikes caused by the notch filter appear.

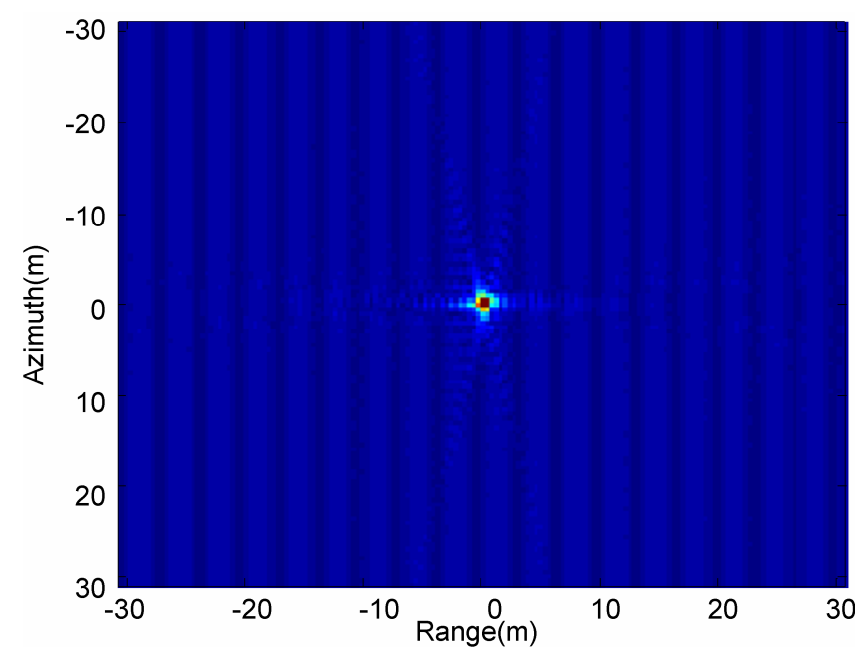

(a)

\section{Imaging}

The statement that the notch filter can suppress RFI but it has negative impact on the matched filter's output has already been demonstrated in the range signal processing. Furthermore, we will demonstrate the theoretical conclusion both in range and azimuth signal processing, that is, in SAR image processing.

Through illuminating the ground with coherent radiation and measuring the echo signals, SAR can produce two dimensional imageries with high resolution of the ground surface. Range resolution is accomplished through range gating. Fine range resolution can be accomplished by using pulse compression techniques. The azimuth resolution depends on antenna size and radar wavelength. Fine azimuth resolution is enhanced by taking advantage of the radar motion in order to synthesize a larger antenna aperture.

The statement for the notch filter being used in SAR processing is also that significantly higher sidelobes would blur the SAR image where RFI signal exist.

SAR image for simulation and experimental UWB SAR data is used to further demonstrate the influence of the notch filter. The procedure of image processing (whatever the simulation or experimental data processing is) is as follows:

1) generating or receiving the radar echo signal mixed with the RFI signal;

2) analysing the echo signal spectrum and identifying the RFI signal, including the RFI signal's carriers and bandwidth;

3) designing the matched filter with notches, as in Equation (14);

4) imaging with the NCS image algorithm [6]. The procedure is illustrated in Figure 13.

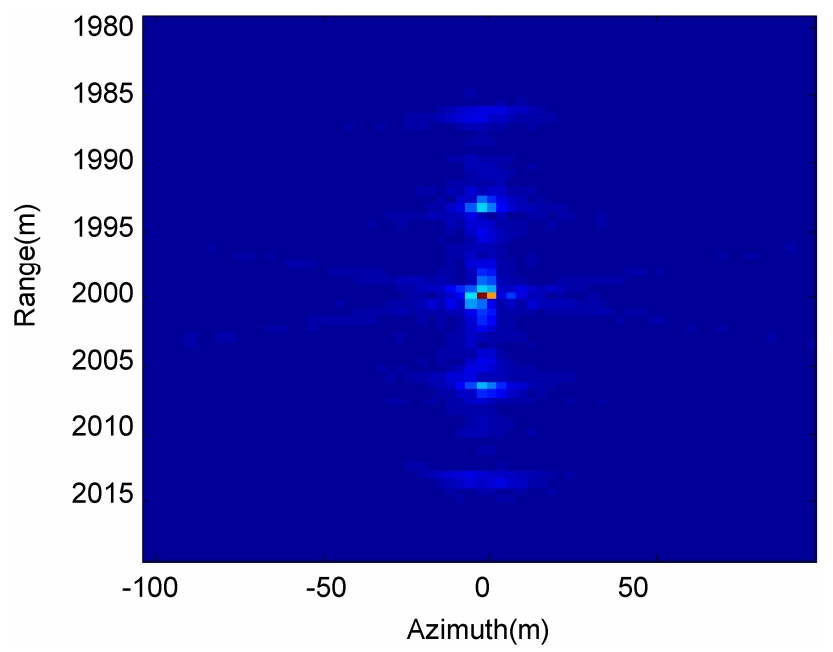

(b)

Figure 14. Imaging for four RFIs with 4MHz bandwidth (a: Without RFI suppression, b: With RFI suppression). 


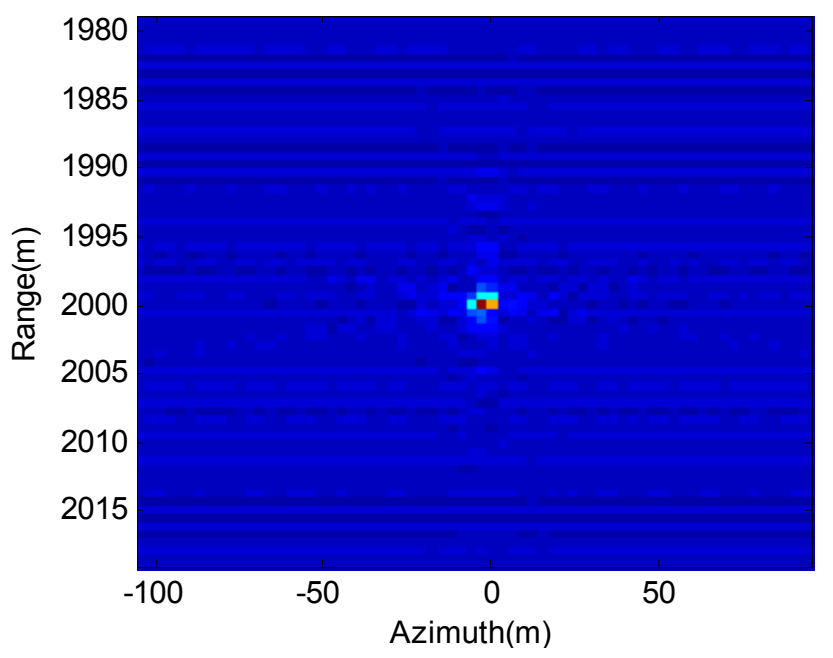

(a)

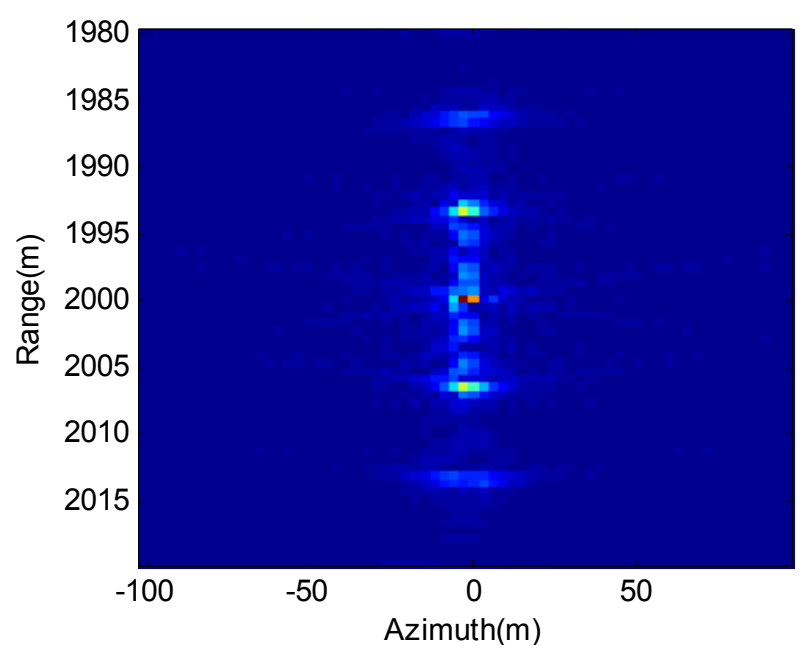

(b)

Figure 15. Imaging for four RFIs with $8 \mathrm{MHz}$ bandwidth (a: Without RFI suppression, b: With RFI suppression).

\subsection{Imaging for Simulation}

In order to study the effect of the notch filter, we have LFM signal with $20 \mathrm{us}$ pulse-width and $200 \mathrm{MHz}$ bandwidth to mix with four RFI signals to form the radar echo.

Firstly, the bandwidths of all the RFI signals are assumed to be the same as $4 \mathrm{MHz}$, and the carriers are $22 \mathrm{MHz}, 44 \mathrm{MHz}, 66 \mathrm{MHz}$ and $88 \mathrm{MHz}$ respectively. After the filter with the four notches and the SAR image is processed, the result is shown in Figure 14. Figure 14(a) is the figure without any RFI suppression, so the regular texture caused by RFI can be seen clearly and Figure 14(b) is the figure with RFI suppression using the notch filter, where the regular texture disappears, but the false targets in range, caused by the notch filter, appear.

Then all the RFI signal bandwidths are assumed to be $8 \mathrm{MHz}$ whereas the other conditions are kept as above. The imaging result is shown in Figure 15. Figure 15(a) is the figure without any RFI suppression and Figure 15(b) is the figure with RFI suppression using the notch filter.

Comparing Figure 14 to Figure 15, we find that RFI certainly blur the SAR image without RFI suppression, whereas the false targets appear in the SAR image with RFI suppression using the notch filter. From the simulation we find that the wider bandwidth the RFI signals have, the stronger the false targets appear in the image. This observation is consistent with the theoretical conclusion.

The influence of the RFI quantities has been studied, and with Figure 14 and Figure 15, the numerical values are measured and described in Table 1 and Table 2, where Table 1 is the result without any RFI suppression and Table 2 is the result with RFI suppression using the notch filter. In the table, the term $(N \times$ RFI with $\mathrm{BMHz})$ means that there are $N$ RFI signals with $\mathrm{BMHz}$ bandwidth in the radar echo.

We draw further conclusions from Table 1 and Table 2, namely that the notch filter has significant effect on sidelobes, as the quantities and the RFI bandwidth increase, the sidelobes rise too. Raised sidelobes result in the SAR image having more false targets.

We also have the conclusion from Table 1 and Table 2 that the notch filter has little effect on the SAR resolution.

\subsection{Imaging for Experimental UWB SAR Data}

The matched filter with notches is applied to experimental UWB SAR data to verify the simulation and theoretical

Table 1. SAR performance without RFI suppression.

\begin{tabular}{|c|c|c|c|}
\hline & Resolution(m) & PSLR(dB) & ISLR(dB) \\
\hline $2 \times$ RFI with $4 \mathrm{MHz}$ & $0.66(\mathrm{r}) \times 0.9(\mathrm{a})$ & -11.32 & -9.71 \\
\hline $2 \times$ RFI with $8 \mathrm{MHz}$ & $0.66(\mathrm{r}) \times 0.9(\mathrm{a})$ & -11.8 & -9.71 \\
\hline $8 \times$ RFI with $4 \mathrm{MHz}$ & $0.66(\mathrm{r}) \times 0.9(\mathrm{a})$ & -11.23 & -9.15 \\
\hline $8 \times$ RFI with $8 \mathrm{MHz}$ & $0.66(\mathrm{r}) \times 0.9(\mathrm{a})$ & -11.59 & -9.74 \\
\hline
\end{tabular}

* Note: ISLR is calculated only in range dimension.

Table 2. SAR performance with RFI suppression.

\begin{tabular}{|c|c|c|c|}
\hline & Resolution(m) & PSLR(dB) & ISLR(dB) \\
\hline $2 \times$ RFI with $4 \mathrm{MHz}$ & $0.66(\mathrm{r}) \times 0.9(\mathrm{a})$ & -11.12 & -6.04 \\
\hline $2 \times$ RFI with $8 \mathrm{MHz}$ & $0.66(\mathrm{r}) \times 0.9(\mathrm{a})$ & -7.84 & -2.15 \\
\hline $8 \times$ RFI with $4 \mathrm{MHz}$ & $0.66(\mathrm{r}) \times 0.9(\mathrm{a})$ & -11.12 & -4.12 \\
\hline $8 \times$ RFI with $8 \mathrm{MHz}$ & $0.66(\mathrm{r}) \times 0.9(\mathrm{a})$ & -9.06 & 1.1 \\
\hline
\end{tabular}

*Note: ISLR is calculated only in range dimension. 
conclusion. The UWB SAR works at UHF frequency band with a bandwidth of $200 \mathrm{MHz}$. Furthermore, The UWB SAR is a subsystem of the multi-frequency-band SAR (MFB SAR) developed in 2005 by the National University of Defense Technology (NUDT) of China, associated with the East China Research Institute of Electronic Engineering (ECRIEE). The MFB SAR system installed on an Y7 aeroplane is capable of operating simultaneously in four frequency bands. Its flight test was performed in January 2005 in an area near the Sanya, Hainan, China. The sky is full of maritime radio and TV signals. Figure 16 is the averaged range signal spectrum of the received base-band signal. This spectrum is real, so we can see that there are at least 6 radio frequency interference signals in the band and their power vary from $10 \mathrm{~dB}$ to $20 \mathrm{~dB}$ greater than the radar echo signal.

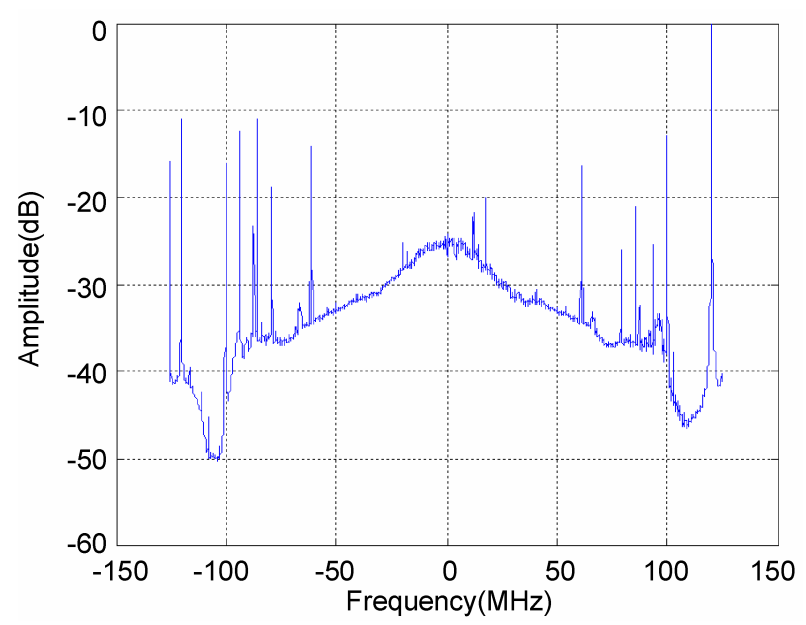

Figure 16. The measure spectrum of RFI.

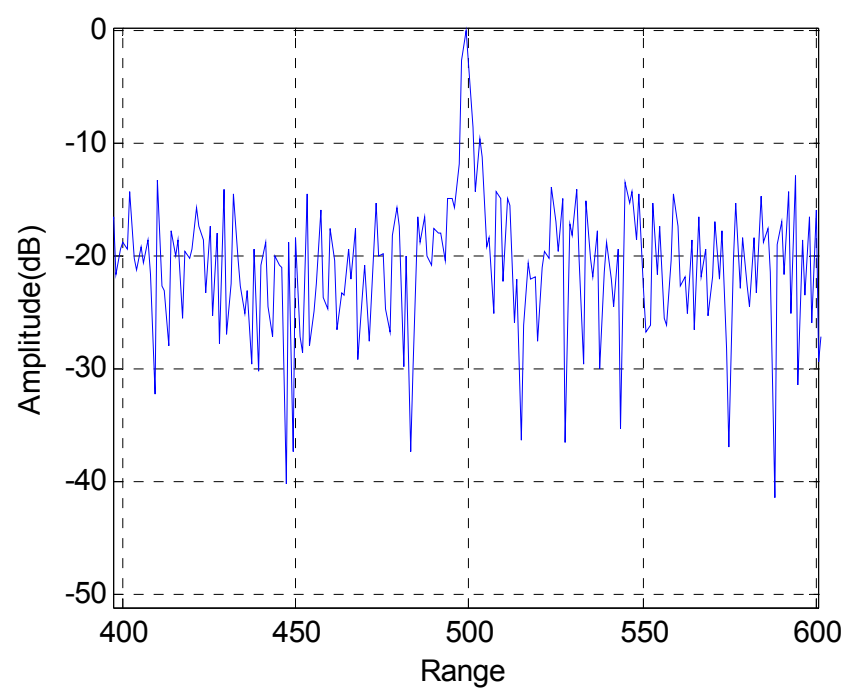

(a)
NCS algorithm is applied to form the UWB SAR image. Figure 17 is a UWB SAR image without RFI suppression. Figure 18 is a cross-section of a point target corresponding to the frame in Figure 17, where Figure 18(a) is the plot along range and Figure 18(b) is the plot along azimuth.

Figure 19 is a UWB SAR image with RFI suppression adopting notch filter. Figure 20 is a cross-section of a point target in the frame of Figure 19, where Figure 20(a) is the plot along range and Figure 20(b) is the plot along azimuth.

We can observe that Figure 17 is blurred with RFI, especially in the relatively dark areas where have low SNR. Meanwhile, Figure 19 has more spots around strong point targets. These spots might be considered targets. But in the dark areas of Figure 19, the contrast of the image clearly improves.

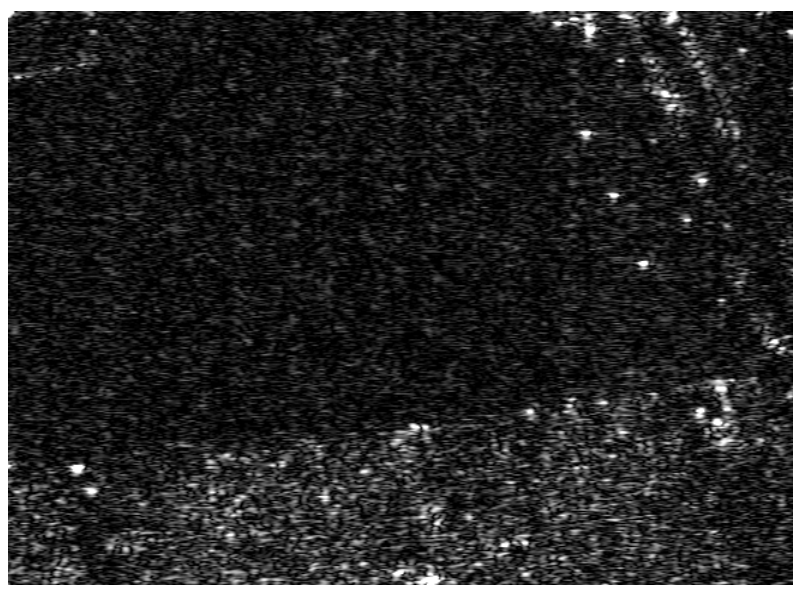

Figure 17. UWB SAR image without RFI suppression.

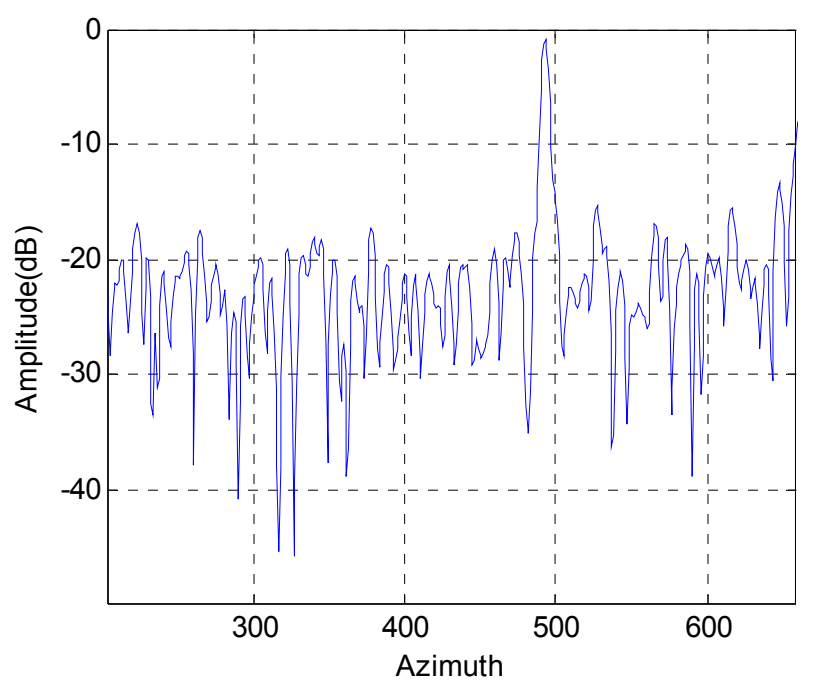

(b)

Figure 18. UWB SAR image without RFI suppression (a: The sectional plot in range, b: The sectional plot in azimuth). 


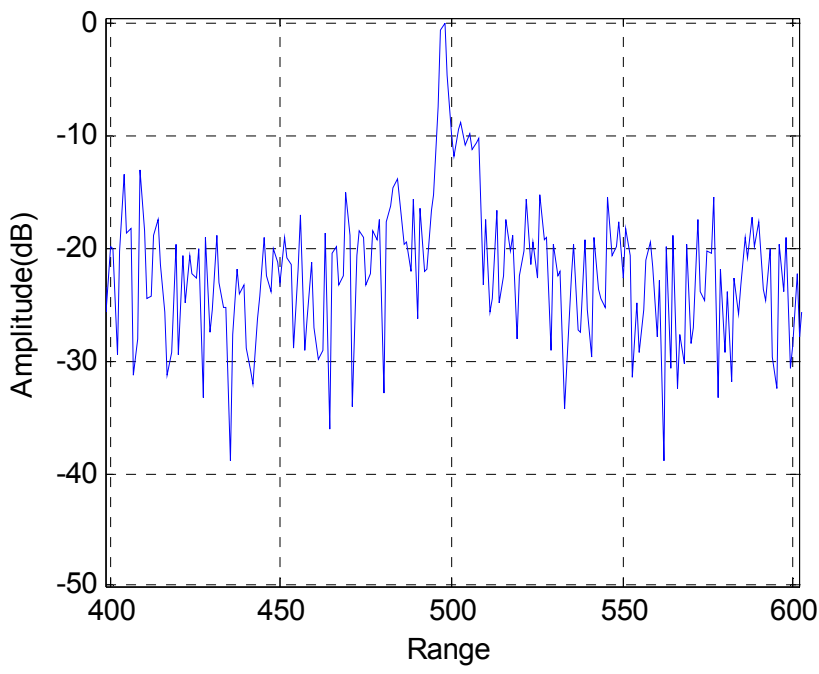

(a)

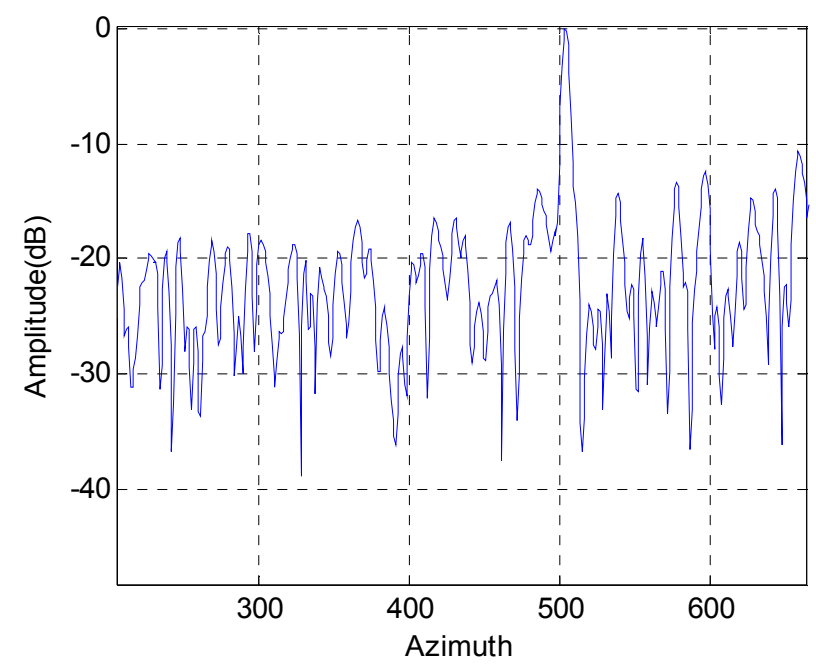

(b)

Figure 20. UWB SAR image without RFI suppression (a: The sectional plot in range, b: The sectional plot in azimuth).

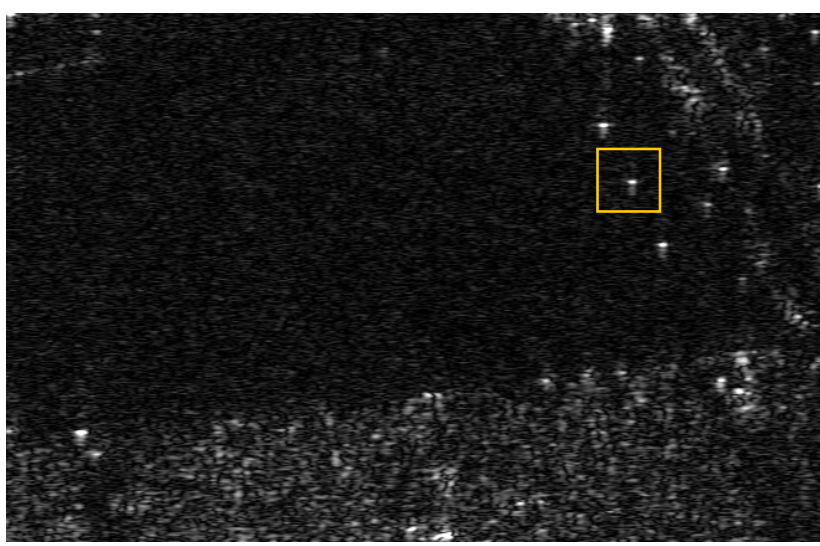

Figure 19. UWB SAR image with RFI suppression.

Table 3. Performance of the UWB SAR image.

\begin{tabular}{|c|c|c|c|}
\hline & Resolution(m) & PSLR(dB) & ISLR(dB) \\
\hline Without RFI suppression & $1.2(\mathrm{r}) \times 2.5(\mathrm{a})$ & -9.88 & -4.79 \\
\hline With RFI suppression & $1.2(\mathrm{r}) \times 2.5(\mathrm{a})$ & -9.38 & -2.98 \\
\hline
\end{tabular}

*Note: ISLR is calculated only in range.

The cross-section of a point target in Figure 18 and Figure 20 is used to analyse the influence before and after RFI suppression. Detailed results are described in Table 3. We find from Figure 18, Figure 19 and Table 3 that: 1) the average power of clutter, especially in range, is reduced after RFI suppression and 2) several spikes appear after RFI suppression adopting notch filter. Fortunately, the notch filter has little impact on the SAR resolution.

\section{Conclusions}

In this paper, the performance of the notch filter is theoretically analysed. Firstly, a matched filter with notch(s) is designed and its theoretical output is derived. The theoretical result shows that the performance of the notch filter is influenced by the notches' width and carrier, and that the notch filter has significant effect on the sidelobes but little effect on the resolution of system impulse response. Secondly, the simulation data and a specified system data are processed in range direction (one-dimension) with notch filter applied to test the validity of the theoretical result. Thirdly, the simulation data and the experimental UWB SAR data are applied to test the validity of the theoretical result. Some useful conclusions are made.

However, despite its shortages the notch filter is an effective tool to suppress RF interference for a small number of narrowband interferers in the lower SNR area of an image.

The theoretical result has been made available to describe the effect of the notch filter on the SAR image. We hope our work and its results may be helpful to who engaged in UWB SAR, wireless system design and RFI suppression research.

\section{References}

[1] T. Koutsoudis and L. Lovas, "RF interference suppression in ultra wideband radar receivers," in Algorithms for Synthetic Aperture Radar Imagery II (D.A. Giglio, ed.), SPIE, Orlando, FL, Vol. 2487, pp. 107-118, April 1995. 
[2] T. Miller, L. Potter, and J. McCorkle, "Army research laboratory RFI suppression for ultra wideband Radar," IEEE Transactions on Aerospace and Electronic Systems, Vol. 33, No. 4, pp. 1142-1156, October 1997.

[3] R. T. Lord and M. R. Inggs, "Efficient RFI suppression in SAR using LMS adaptive filter integrated with range/ Doppler algorithm," Electronics Letters, Vol. 35 No. 8, pp. 629-630, April 15, 1999.

[4] R. T. Lord and M. R. Inggs, "Approaches to RF interference suppression for VHF/UHF synthetic aperture radar,"
Communications and Signal Processing, COMSIG'98, pp. 95-100, 1998.

[5] X. Luo, L. M. H. Ulander, J. Askne, G. Smith and P. O. Frolind, "RFI suppression in ultra-wideband SAR systems using LMS filters in frequency domain," Electronics Letters, Vol. 37, No. 4, February 15, 2007.

[6] G. W. Davidson, I. G. Cumming, and M. R. ITO, “A chirp scaling approach for processing squint mode SAR data," IEEE Transactions on Aerospace Electronic Systems, Vol. 32, pp. 121-133, 1996. 